\title{
Light collection and wavelength calibration for an extreme ultraviolet diode array spectrograph
}

\author{
M. C. Marconi, J.F. Schmerge, and J.J. Rocca \\ Electrical Engineering Department, Colorado State University, Fort Collins, Colorado 80526
}

(Received 19 September 1988; accepted for publication 3 January 1989)

\begin{abstract}
We describe the use of a simple spherical aluminum-coated mirror to image the radiation of a cistant XUV-emitting plasma in the slit of a vacuum spectrograph. Calculations to determine the optimum position and curvature radius of the mirror as a function of the divergence of the emitted plasma radiation are described. Efficient light collection by the grazing incidence mirror allows calibration of an intensified diode array used as a detector with a low-pressure ( $\left.10^{-4} \mathrm{Torr}\right) \mathrm{dc}$ capillary discharge that does not require a complex differential pumping system.
\end{abstract}

The spectroscopic study of the light emitted by a plasma is a powerful tool to determine its physical characteristics. Important data from dense plasmas can be obtained at extreme ultraviolet (XUV) wavelengths where the plasma is generally optically thin allowing the recording of well-defined line spectra. However, working in this spectral region requires the use of experimental procedures that complicate the detection setup compared with visible light spectroscopy. For example, optical windows are not available and lenses cannot be used to focus the radiation. ${ }^{1,2}$ Mirror systems are commercially available to increase the light gathering capabilities of XUV spectrographs but they are relatively expensive. ${ }^{3}$

Here, we describe the use of an inexpensive spherical aluminum-coated mirror to image the divergent extreme ultraviolet radiation from a plasma source into the slit of a vacuum spectrograph. The position and width of the produced image depends strongly on the divergence of the radiation produced by the source. It also depends on the curvature radius of the mirror, the distance between the mirror and the source, and the grazing incidence angle. We describe a program developed to calculate, from geometrical considerations, the image position obtained by a spherical mirror from a divergent source taking into account the incidence angle and the curvature radius of the mirror. The program also allows the calculation of the required radius of curvature for the mirror, considering that the distance between the plasma source and the spectrometer is fixed. The experimental implementation and the results obtained are also discussed.

We have recently proposed the plasma of highly ionized capillary discharges as a laser source in the XUV and soft $x$ ray. ${ }^{4}$ Capillary discharges can create dense highly ionized plasmas with relatively modest excitation energy. ${ }^{5}$ Rapid plasma cooling occurring at the decay of the discharge pulse is expected to result in large electron-ion recombination rates and the possibility of optical gain at short wavelengths. To study the capillary plasma and the possibility of XUV amplification, we are using a $1-\mathrm{m}$ normal incidence $f=10.4$ vacuum spectrograph in order to analyze the emitted light in the VUV and XUV region. The detector used in the spectrograph is an optical multichannel analyzer (OMA) com- posed of two multichannel plate intensifiers stacked in front of a 1024 photodiode array. The detector is similar to other windowless multichannel plate array detectors described in the literature ${ }^{6,7}$ but with the difference that it contains two close-spaced multichannel plates for increasing the sensitivity. The front surface of the first multichannel plate, which faces the light, is coated with copper iodide for increased VUV and XUV response. ${ }^{8} \mathrm{~A}$ second multichannel plate is placed behind the front plate to achieve additional electron multiplication and a gain as high as $10^{7} .9$ Electrons emerging from the second plate are accelerated towards a phosphorus screen where they produce optical radiation which is guided by a fiber optic bundle and detected by the photodiode array. The multichannel plate intensifier was provided by Galileo Electro Optics (Sturbridge, MA) and interfaced to an array of $25-\mu \mathrm{m}$ diodes manufactured by Princeton Instruments. The detector is placed in the focal plane of the spectrograph allowing the simultaneous recording of a spectrum $420 \AA$ wide, using a 600 -lines-per-mm grating. The resolution of the system with this grating is $0.41 \AA$ per channel.

The windowless OMA is connected to a personal computer which digitalizes and stores the acquired data. The detection system involves electronics which should be protected from the electromagnetic noise produced by the fast high-current discharge, so the entire detection system is located inside a Faraday enclosure. The spectrograph is also located inside this electromagnetic shield, and its entrance slit is located more than $1-\mathrm{m}$ from the plasma source. Consequently, light collection for an entrance slit width of the order of $20 \mu \mathrm{m}$ is small, and some kind of focusing device is necessary to increase the signal.

A scheme of the light collection system we implemented is shown in Fig. 1. Light collection by the spherical grazing incidence mirror is very efficient, producing a vertical linear image that matches the vertical entrance slit of the spectrograph. The use of the focusing mirror has the extra advantage of stopping the particles emitted by the expanding plasma protecting in this way the expensive spectrograph diffraction grating. This avoids the use of complex fast shutters to stop the plasma particies. ${ }^{10}$ The relatively inexpensive mirror car be cleaned or eventually replaced if damage occurs by particle deposition. 

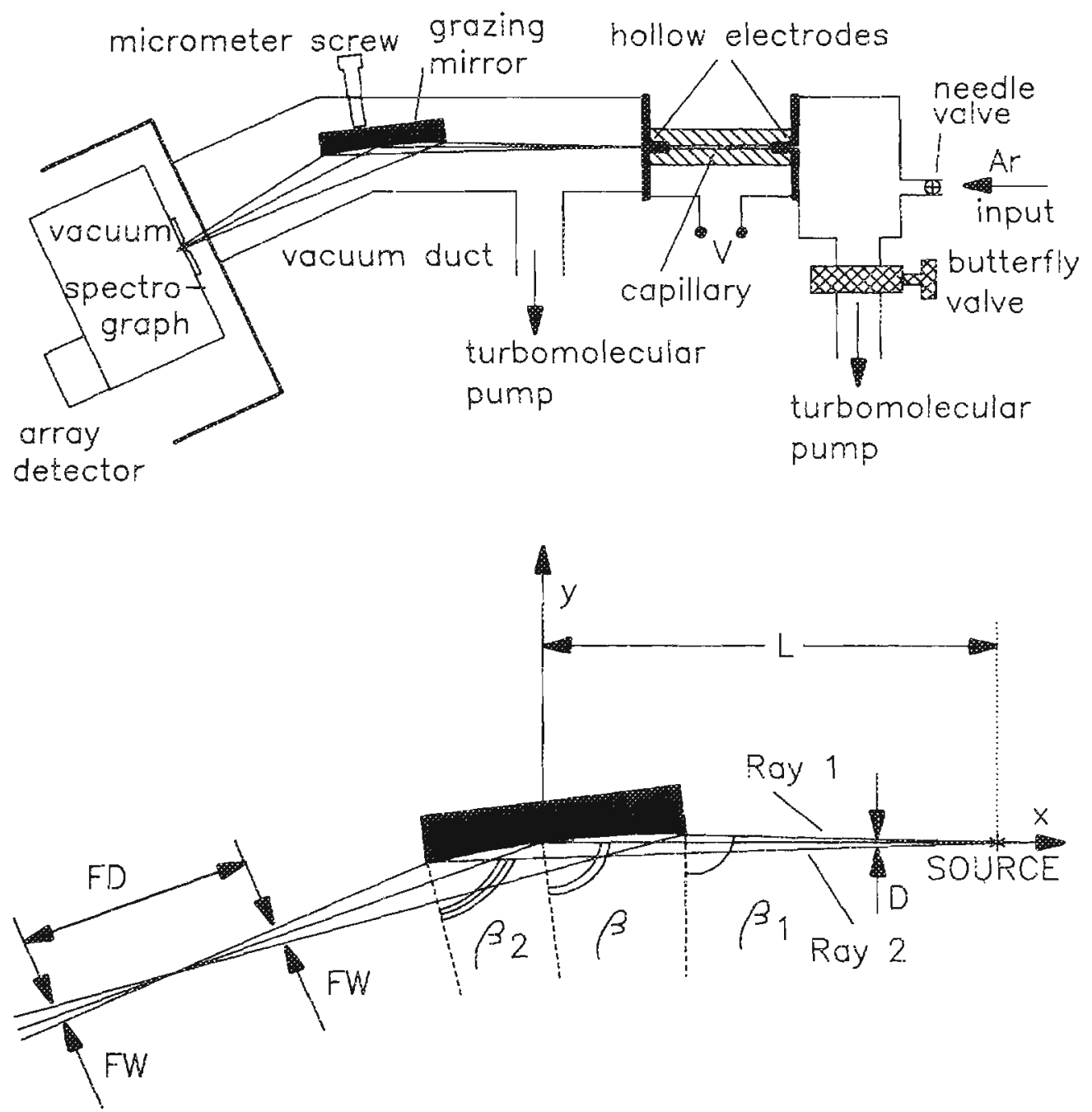

Fig. 1. (a) Experimental setup showing the grazing incidence mirror used to image the radiation in the spectrograph siit. (b) Detail of the mirror assembly showing the parameters used in the code listed in Appendix A.
For design calculations purposes in the case of a capillary plasma, we considered the divergence of our source as the average value obtained by calculating the solid angie stibtended from the center of the capillary. For our purposes this is a good approximation of the divergence of the incoherent plasma radiation. The code listed in Appendix $\mathrm{A}^{11}$ calculates the path of the two extreme beams incident on the mirror edges and the central beam [Fig. 1(b)]. Working in grazing incidence, the mirror does not perfectly focus these three beams at the same point. However, the deviation from a perfect focus can be neglected since it is tsually smail. Nonetheless, the program estimates the image sharpness and calculates the focal depth. From the three calculated paths, the program determines the locations where the image has a given transverse size. The values obtained with the program were experimentally verified within a measurement error of less than 5\% using a $\mathrm{H}$ eNe plasma tube as the incoherent light divergent source. This piasma has a divergence similar to cur capillary discharge.

We used the code to design the light collection system for our vacuum spectrograph located at $1.41 \mathrm{~m}$ from a 500 $\mu \mathrm{m}$-diam $40-\mathrm{mm}$-long capillary discharge. After running the code we selected for our experiment a 3-m-curvature radius aluminum-coated mirror of $1.5 \mathrm{in}$. diam placed at 56 $\mathrm{cm}$ from the discharge and giving an image at $85 \mathrm{~cm}$ from the mirror. The image in this case has a width less than $100 \mu \mathrm{m}$ within a $0.6-\mathrm{cm}-\mathrm{region}$ centered in the focal point. The mirror was placed vacuum-mounted in a holder which allows adjustment of the incidence angle. The vacuum duct is fixed in such a way that the grazing angle on the mirror is $13^{\circ}$ [ that gives a reflectivity for aluminum better than $60 \%$ for wavelengths as low as $530 \AA$ (Ref. 12)]. A micrometer screw permits, through a vacuum feedthrough, the final adjustment of the angle, thereby fixing the final image position on the spectrograph siit. Easy alignment of the system is achieved by visually observing the relative position of the focal line of the visibie radiation refiected by the mirror with respect to the spectrograph slit through a window built in front of the spectrograph for that purpose.

The efficient light collection by the mirror allows the wavelength calibration of the instrument using a low-pressure low-current de capillary discharge. This low-pressure discharge produces enough light to calibrate the system. In comparison with previously used high-pressure capillary discharges, the low-pressure discharge has the advantage that no differential pumping other than the one provided by the discharge capillary and spectrograph slit itself is necessary allowing a pressure of $1 \times 10^{-4}$ Torr in the capillary region and at the same time a pressure of $1 \times 10^{-6}$ Torr in the spectrograph. In this regime a de discharge can be estab- 
lished in the capilary by applying $15 \mathrm{kV}$ between the electrodes. For calibration purposes we used a $20-\mathrm{mA}$ argon discharge through a $500-\mu \mathrm{m}$, 4-crn capillary. Two hollow graphite electrodes are positioned at each end of the capillary allowing a clear optical path for light collection. The capillary was pumped from both ends using a $400-\ell / \mathrm{s}$ turbomolecular pump. Argon gas is admitted through a needle valve to the capillary end opposite the spectrograph and is pumped through the discharge channel in such a way that a continuous flux of argon can be established in the capillary. The Ar spectra allow a very easy and accurate calibration of the system in the VUV and XUV with no extra equipment than the discharge setup. Other gases can also be used to obtain other calibration spectra at different wavelengths if necessary. A typical extreme ultraviolet calibration spectrum obtained is presented in Fig. 2 where 18 lines of ArII and Arul can be easily identified, permitting a precise calibration of the detector array spectrograph. To obtain these spectra the back face of the second multichannel piate, which faces the phosphorus screen, is grounded and the screen is positively biased to $4 \mathrm{kV}$ with a dc power supply. The front face of the second plate is biased negatively to $-850 \mathrm{~V}$ and $-800 \mathrm{~V}$ is applied across the first multichannel plate. The main advantage of this calibration method is the simplicity, and in our case the fact that the same capillary structure that is the subject of our present study is used in the calibration procedure allowing an in situ wavelength calibration and a good optical alignment of the system. If required, an absolute intensity calibration in the extreme ultraviolet could be obtained by the method of branching ratios of visible and XUV transitions having the same upper level using a calibrated visible monochromator to measure the intensity of the radiation emitted through the other end of the capillary.

The high light-collection efficiency of the spherical mirror allows time-resolved single shot XUV spectra of the emission of a short pulse ( 50 ns FWHM) capillary discharge excited with only a few Joules of energy. A time-resolved single shot spectrum from the axial emission of a lithium hydrite capillary discharge $500 \mu \mathrm{m}$ diam and $40 \mathrm{~mm}$ in length excited by a $5 \mathrm{~J}$ current pulse is shown in Fig. 3. The spectrum was obtained by gating the gain of the front multichannel plate with a 15 -ns pulse centered at 23 ns after the peak current of a 50 ns FWHM, 5-kA discharge current pulse. To record these spectra, a negative bias of 780 and 180 $\mathrm{V}$ were applied across the second and first multichannel plate, respectively. With this low bias voltage across the first plate the gain of the detector is very low, and no light is detected. Switching of the gain is achieved by increasing the voltage drop across this plate by applying an additional

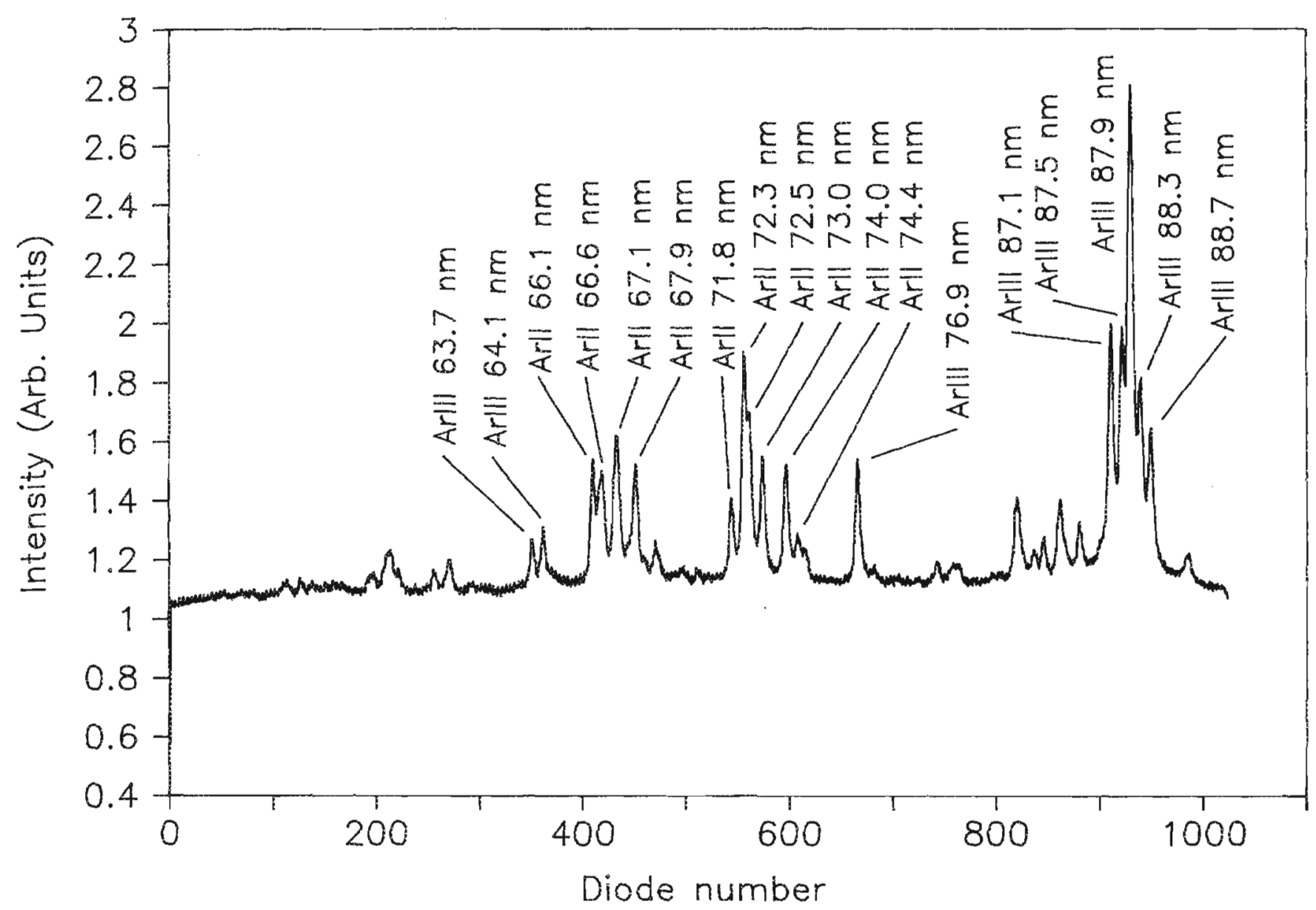

Fig. 2. Extreme ultraviolet calibration spectrum obtaincd from a low-pressure de Ar capillary discharge. 


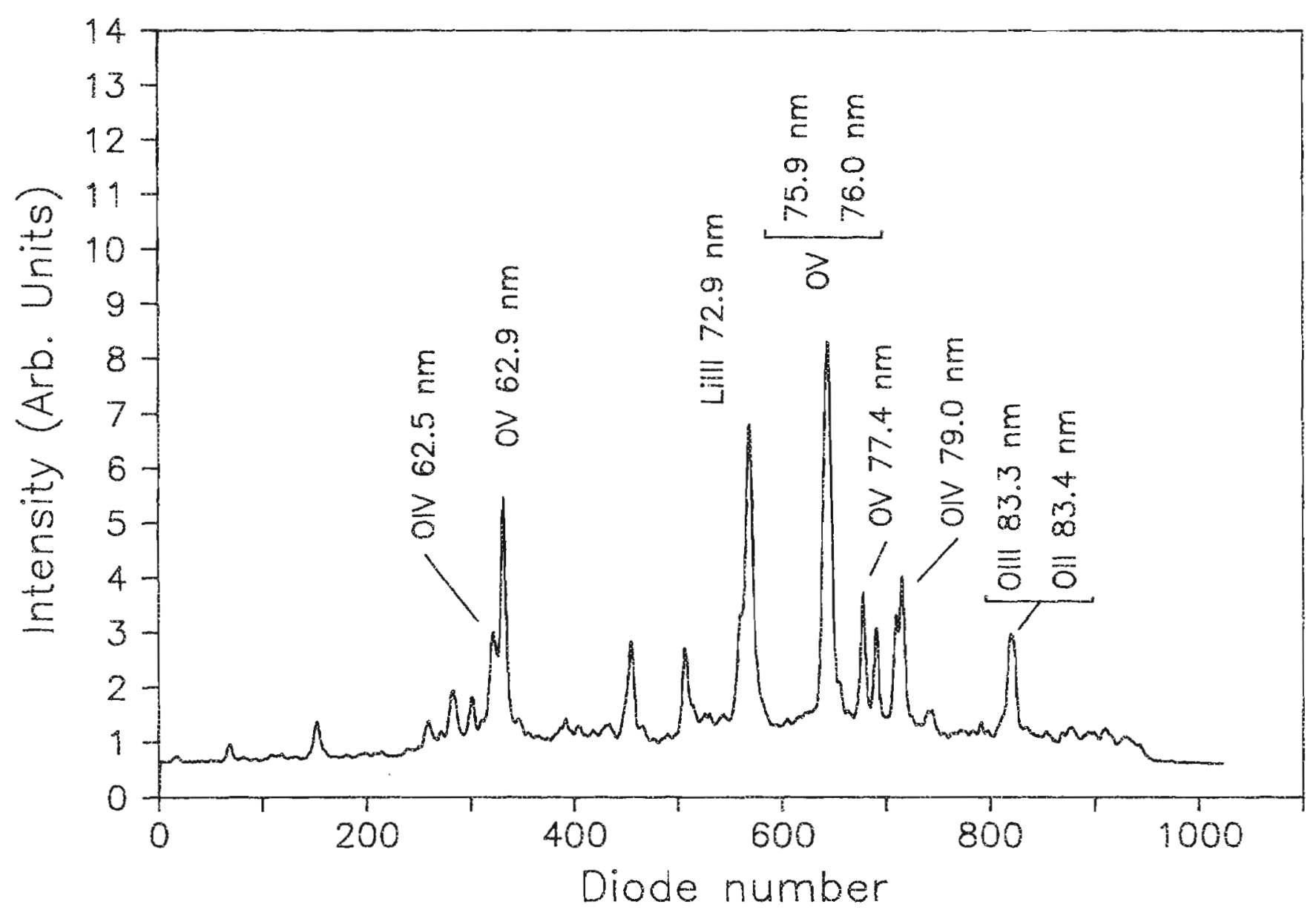

FiG. 3. Time-resolved single shot XUV spectrum from the axial emission of a LiH capillary discharge.

$-500-\mathrm{V}$ puise. This scheme has the advantage of reducing the voltage requirements of the pulser to $500 \mathrm{~V}$ allowing its implementation by discharging a transmission line through a commercially available switch. The switch that discharges the transmission line into the plate uses a single MOSFET and is manufactured by Directed Energy (Fort Collins, CO).${ }^{13}$ The length of the transmission line determines the width of the pulse, which in our experiment was $15 \mathrm{~ns}$. The Lin $729-\AA$ line and multiple ionized oxygen impurity lines are easily identified based on the Ar calibration spectra of Fig. 2.

This work was supported by the National Science Foundation Grant No. ECS 8606226. M. C. Marconi was supported by a fellowship from Universidad Nacional de Buenos Aires and Consejo Nacional de Investigaciones Cientificas y Tecnicas de la Republica Argentina. We acknowledge George J. Krausse from Directed Energy Inc. for his valuable assistance with the construction of the fast highvoltage pulser used to gate the multichannel plate and $\mathrm{Y}$. Talmi from Princeton Instruments for fruitfui discussions. J.J.R. is a National Science Foundation Presidential Young Investigator.
'A. N. Zaidel and E. Ya. Shreider, Vacuum Ultraviolet Spectroscopy (Humphrey Science, London, 1970).

2y. A. R. Samson, Technigues of Vacium Ultraviolet Spectroscopy (Wiley, New York. 1967).

"Acton Research Corporation Catalog, Box 215, 525 Main Street, Acton, MA 01720.

4J. J. Roeca, D. C. Beethe, and M. C. Marconi, Opt. Lett. 13, 565 (1988).

'P. Bogen, H. Conrands, G. Gatti, and W. Kohlhaas, J. Opt. Soc. Am. 58, 2

(1968); R. A. McCorkle, Appl. Phys. A 26, 261 (1981).

'T. Fonk, A. T. Ramsey, and R. V. Yelle, Appl. Opt. 21, 2115 (1982).

${ }^{7}$ D. F. Register, G. L. Jackson, and S. E. Walker, in 5 th Topical Conference on High Temperature Plasma Diagnostics, edited by E. M. Campbell and G. W. Leppelmeier (AIP, New York, 1985).

${ }^{\gamma}$ R. K. Richardis, Rev. Sci. Instrum. 49, 1210 (1978).

${ }^{9} J$. I. Wiza, Nucl. Instrum. Methods 162, 587 (1979).

${ }^{16}$ P. R. A. Lyons, D. Kilpin, and S. L. Gai, I. Phys. E 17, 108 (1984).

"See AIP document Nu. PAPS RSINA-60-966-02 for 2 page of Appendix A computer program. Order by PAPS number and journal reference from American Institute of Physics, Physies Auxiliary Publication Service, 335 East 45th Strect, New York, NY 10017. The price is $\$ 1.50$ for each microfiche ( 98 pages) or $\$ 5.00$ for photocopies of up to 30 pages, and $\$ 0.15$ for each additional page over 30 pages. Airmail additional. Make checks payable to American Institure of Physics.

12R. F. Malina and W. Cash, Appl. Opt. 17, 3309 (1978).

${ }^{13}$ FPS3N Data Sheet, Directed Encrgy, 2301 Research Boulevard, Fort Collins, CO 80526. 